\title{
Clinical and Histopathological Characteristics of Psoriasis in Dr. Hasan Sadikin General Hospital Bandung from 2009 to 2013
}

\author{
Willy Chandra Hermawan, ${ }^{1}$ Hermin Aminah, ${ }^{2}$ Dendi Sandiono ${ }^{3}$ \\ ${ }^{1}$ Faculty of Medicine, Universitas Padjadjaran, ${ }^{2}$ Department of Pathological Anatomy Faculty of \\ Medicine Universitas Padjadjaran/Dr. Hasan Sadikin General Hospital Bandung, ${ }^{3}$ Department of \\ Dermato-Venereology Faculty of Medicine Universitas Padjadjaran/Dr. Hasan Sadikin General \\ Hospital Bandung
}

\begin{abstract}
Background: Psoriasis is a chronic inflammatory skin disease characterized by alterations in epidermal growth and differentiation. Its primary cause remains unknown, and its clinical and histopathological characteristics sometimes change from time to time. The study aimed to reveal the clinical and histopathological characteristics among psoriasis patients in Dr. Hasan Sadikin General Hospital Bandung. Methods: This study involved 162 medical records of psoriasis patients who visited the Department of Dermato-Venereology and 40 medical records of psoriasis patients who visited the Department of Pathological Anatomy of Dr. Hasan Sadikin Hospital, from 2009 to 2013. Medical record data were collected to describe the patient's clinical and histopathological characteristics of psoriasis. The collected data were analyzed by frequency distribution.

Results: From the clinical medical records, the characteristics found among the psoriasis patients were: $14(8.6 \%)$ having familial history, $160(98.8 \%)$ having reddish plaque, $155(95.7 \%)$ having silvery scale, $40(24.7 \%)$ having pustules, 6 (3.7\%) having positive Auspitz sign, 48 (29.6\%) having nail involvement, and $20(12.4 \%)$ having tongue involvement. In addition, from the histopathological medical records, the characteristics of epidermis among the psoriasis patients found were: $36(90 \%)$ having hyperkeratosis with parakeratosis, 39 (98\%) having rete ridge elongation and/or acanthosis, 35 (88\%) having Munro's microabcess and/or micropustules of Kogoj, 15 (38\%) having thin stratum granulosum, and 30 (75\%) having blood vessels dilatation.

Conclusions: In psoriasis, the most common clinical characteristics are reddish plaque while the most common histopathological characteristics are elongation of the rete ridges and/or acanthosis. [AMJ.2016;3(4):556-61]
\end{abstract}

Keywords: Clinical characteristics, histopathological characteristics, psoriasis

\section{Introduction}

Psoriasis is a chronic inflammatory skin disease with abnormal epidermal growth and differentiation characteristics. It is indicated by an increasing of proliferation and a shortening of keratinocyte maturation period. The exact cause of psoriasis has not been yet defined. ${ }^{1}$ Based on its clinical characteristics, psoriasis can be further categorized as psoriasis vulgaris, sebopsoriasis, pustular psoriasis, guttate psoriasis, and erythrodermic psoriasis. ${ }^{1}$

The onset of psoriasis has a close link to family history, especially in early onset psoriasis patients. Previous studies did not find any significant difference in the disease prevalence between genders. ${ }^{2}$

Clinical and histopathological characteristics are derived from inflammation, which causes hyperproliferation and a failure in keratinocyte differentiation. ${ }^{3}$ Hyperproliferation in histopathological characteristics affects hyperkeratosis with parakeratosis, the thinning of stratum granulosum, and rete ridge elongation and/or acanthosis; moreover, in clinical characteristics, it causes plaque and scales on the skin. The plaque is reddish in color due to vasodilation. ${ }^{4}$ Newly formed blood vessels

Correspondence: Willy Chandra Hermawan, Faculty of Medicine, Universitas Padjadjaran, Jalan Raya BandungSumedang Km.21, Jatinangor, Sumedang, Indonesia, Phone: +628112036688 Email: willychermawan@gmail.com 
are easily damaged, resulting in bleeding if the overlying skin is detached (Auspitz sign). ${ }^{5}$ When neutrophils collect in the stratum corneum, it is called Munro's microabcess. It is called Kogoj's micropustules when they collect in the stratum spinosum. ${ }^{6}$ Nails and tongue, both having cells derived from keratinocytes, can also be affected. ${ }^{4}$ This study aimed to examine the clinical and histopathological characteristics of psoriasis patients.

\section{Methods}

This was a descriptive study. The data were collected retrospectively from secondary data in the form of patients' medical records in the Department of Dermato-Venereology and Department of Pathological Anatomy Dr. Hasan Sadikin General Hospital Bandung from 1 January 2009 to 31 December 2013 (5year data). The aim and methods of this study had been approved by ethics committee of Dr. Hasan Sadikin General Hospital Bandung. The samples used in this study were selected by total sampling (162 clinical and 40 histopathological medical records).

The samples in this study were all medical records from the patients first visit that were clinically or histopathologically diagnosed psoriasis. Psoriasis patients who had got previous medical care were excluded.

The characteristics examined in this study were the clinical characteristics including age, gender, types of psoriasis, family history, lesion distribution, reddish plaque, silvery scales, pustules, Auspitz sign, nail involvement, and tongue involvement; besides, the histopathological characteristics were including hyperkeratosis with parakeratosis, rete ridge elongation and/ or acanthosis, Munro's microabcess and/or Kogoj's micropustules, thinning or depletion of stratum granulosum, and dilatation of blood vessels.

The collected data were calculated using frequency tabulation and percentage.

\section{Results}

Based on the inclusion criteria, a total of 162 cases from clinical medical records were included in this study. From this cases which were diagnosed clinically as psoriasis, 46 cases underwent biopsies and the samples were sent for histopathological examination. From the 46 cases, 33 cases were diagnosed psoriasis based on histopathology and 13 cases were not psoriasis. There were 7 cases which were diagnosed psoriasis based on histopathology but were not sent with psoriasis clinical diagnosis. As a result, a total of 40 medical records from histopathology were diagnosed psoriasis.

The mean age of patients who were the subjects of this study is 39.7 years old. The gender of patients showed relatively proportionate comparison, in which 90 (56\%) cases were females and 72 (44\%) were males, with proportion of 1.25 for female to male cases.

Psoriasis vulgaris was the most commonly diagnosed type of psoriasis, with its proportion exceeding half of total cases of psoriasis

\section{Table 1 Type and Lesion Distribution of Psoriasis}

\begin{tabular}{lc}
\hline \multicolumn{1}{c}{ Clinical Characteristics } & $\mathbf{n ( \% )}$ \\
\hline Psoriasis Type & \\
Psoriasis Vulgaris & $88(54.4 \%)$ \\
Sebopsoriasis & $16(9.9 \%)$ \\
Pustular Psoriasis & $29(17.9 \%)$ \\
Guttate Psoriasis & $7(4.3 \%)$ \\
Erythrodermic Psoriasis & \\
Others & $2(1.2 \%)$ \\
Lesion Distribution & \\
Regional & $77(47.5 \%)$ \\
Generalized & $63(38.9 \%)$ \\
Universal & $22(13.6 \%)$ \\
\hline
\end{tabular}


Table 2 Clinical Characteristics of Psoriasis

\begin{tabular}{lccc}
\hline \multicolumn{1}{c}{ Clinical Characteristics } & Positive & Negative & Not Stated \\
\hline Family History & $14(8.6 \%)$ & $84(51.9 \%)$ & $64(39.6 \%)$ \\
Reddish Plaque & $160(98.8 \%)$ & $2(1.2 \%)$ & $0(0 \%)$ \\
Silvery Scale & $155(95.7 \%)$ & $7(4.3 \%)$ & $0(0 \%)$ \\
Pustule & $40(24.7 \%)$ & $0(0 \%)$ & $122(75.3 \%)$ \\
Auspitz Sign & $6(3,7 \%)$ & $0(0 \%)$ & $156(96.3 \%)$ \\
Nail Involvement & $48(29.6 \%)$ & $46(28.4 \%)$ & $68(42 \%)$ \\
Tongue Involvement & $20(12.4 \%)$ & $59(36.4 \%)$ & $83(51.2 \%)$ \\
\hline
\end{tabular}

(54.4\%). The most common distribution of lesion was regional distribution (Table 1).

The most common clinical characteristics of psoriasis were reddish plaques (98.8\%) and silvery scales (95.7\%) (Table 2).

The most common histopathological characteristics were elongation of rete ridge and/or acanthosis which were found in 98\% of cases, followed by hyperkeratosis with parakeratosis which were found in $90 \%$ of cases (Table 3).

\section{Discussions}

Psoriasis has close link to genetic factors, and the gene that has been considered important in the development of psoriasis is HLA-Cw6. This gene encodes the surface proteins of antigen presenting cell to be presented to CD8+ T-cells, the dominant type of white blood cells in psoriatic lesions. ${ }^{1}$ The mean age of onset in this study was 39.7 years; it is higher than Griffith's finding, which is 32 years. ${ }^{4}$ This can be explained by Stuart's theory which states that a younger age of onset (under 40 years) has a close relationship with hereditary aspect of HLA-Cw6 gene. ${ }^{7}$ In this study, the risk factor of having HLA-Cw6 was probably low, as indicated by the rate of family history of similar diagnosis or symptoms $(8.6 \%)$ that was even lower than a previous study by Altobelli et al. ${ }^{8}$ in Italy (45.9\%) although there were many cases of unknown family history due to incomplete anamnesis (39.6\%). This was also supported by a study from Jin et al. ${ }^{9}$ which discovers that HLA-Cw6 gene is less prevalent in Asian populations than in European populations.

In this study, the proportion of gender was relatively proportionate, which was 1.25 . This conformed to the finding of Augustin et al. ${ }^{10}$ which describes the ratio of the female and male psoriasis case prevalence was close to 1 .

A previous study by Griffith and Barker ${ }^{4}$ discovers psoriasis vulgaris to be the type with the largest proportion, which was about $80 \%$. Psoriasis vulgaris has clinical presentations of reddish plaques with silvery scales. ${ }^{1}$ In this study, psoriasis vulgaris was also the most frequent type, but with a smaller proportion (54.5\%). This was due to the higher proportions of the other types of psoriasis compared to the findings of Griffith and Barker. ${ }^{4}$ The type with a higher proportion than the one described by Griffith and Barker ${ }^{4}$ was generalized pustular psoriasis (van Zombusch) (15\%) and erythrodermic psoriasis $(12.3 \%)$. Generalized pustular psoriasis has a clinical presentation of generalized or universal reddish plaques, with overlying silvery scales and pustules. Erythrodermic psoriasis has a

Table 3 Histopathological Characteristics of Psoriasis

\begin{tabular}{lccc}
\hline \multicolumn{1}{c}{ Histopathological Characteristics } & Positive & Negative & Not Stated \\
\hline Hyperkeratosis with Parakeratosis & $36(90 \%)$ & $0(0 \%)$ & $4(10 \%)$ \\
Rete Ridge Elongation / Acanthosis & $39(98 \%)$ & $0(0 \%)$ & $1(2 \%)$ \\
Kogoj's / Munro's Sign(s) & $35(88 \%)$ & $1(2 \%)$ & $4(10 \%)$ \\
Lost / Thinning of Stratum Granulosum & $15(37 \%)$ & $0(0 \%)$ & $25(63 \%)$ \\
Blood Vessels Dilatation & $30(75 \%)$ & $0(0 \%)$ & $10(25 \%)$ \\
\hline
\end{tabular}


clinical presentation of reddish plaques or macules which cover at least $90 \%$ of the total skin surface. ${ }^{4}$ Generalized pustular psoriasis and erythrodermic psoriasis were types that have clear precipitating factors, which are infections, unprescribed corticosteroid use, and irrational use of cream medication. ${ }^{1,11}$

Regional distribution (47.5\%) was the most frequent type of distribution, with main predilections in the elbows and knees. Such predilections can be attributed to the frequent physical contact in those parts of the body (Koebner phenomenon). ${ }^{1,4}$ Generalized (38.9\%) and universal (13.6\%) types had quite high proportions, probably due to the high frequency of generalized pustular and erythrodermic psoriasis in this study.

Psoriasis is included as papulosquamous dermatitis, which is the inflammation of the skin with epidermal hyperkeratosis and desquamation. The diseases of this condition include seborrheic dermatitis, lichen planus, pityriasis rosea, and tinea corporis. The clinical presentation of psoriasis often resembles other papulosquamous dermatitis diseases, and hence requires histopathological examination, especially in psoriasis with atypical presentation, such as sobopsoriasis, pustular psoriasis, dan erythrodermic psoriasis. ${ }^{1,4}$

Reddish plaque is an elevated skin lesion, with a diameter larger than $0.5 \mathrm{~cm}$, which is reddish in color. Silvery scales is defined as the presence of detached outer skin tissue which results in rough and silvery skin surface. Pustules are elevated skin lesions with pus content. ${ }^{12}$ In this study, reddish plaques were found in 160 cases $(98.8 \%)$ and silver scales in 155 cases $(95.7 \%)$. This was in line with Griffith and Barker ${ }^{4}$, who states reddish plaques and silvery scales are as the most representative characteristics of psoriasis. Reddish plaques and silvery scales are caused by keratinocyte hyperproliferation in the epidermis overlying dilated and tortuous blood vessels. ${ }^{1}$ Pustules were found in 40 patients $(24.7 \%)$, which was higher than the one reported by Griffith and Barker. ${ }^{4}$ This was due to the high proportion of pustular psoriasis in this study (17.9\%).

Auspitz sign is a characteristic clinical manifestation of psoriasis that is rarely found among other papulosquamous dermatitis conditions. Auspitz sign is the bleeding that occurs when the scaly skin in psoriatic lesion is detached. This is because the newly formed blood vessels are fragile and because of the thinning of stratum granulosum. Both processes are due to the inflammatory patterns of psoriasis that involves IFN- $\gamma$, IL-2, IL-6, and TNF- $\alpha$, which stimulate hyperproliferaiton of keratinocytes and hence disrupt the differentiation of stratum granulosum and endothelial differentiation which result in the formation of new, yet fragile and tortuous blood vessels., ${ }^{1,413}$ In this study, Auspitz sign examination was only performed on 6 cases (3.7\%). The very few number of Auspitz examination was probably due to the fact that this examination inflicts pain on the patient. Patients who had Auspitz sign examination were all inpatients and recommended to proceed to histopathological examination. This indicated that Auspitz sign examination was treated on patients whose diagnosis had not been able to be confirmed as psoriasis.

Beside the skin, psoriasis can also involve the nails and tongue which have keratinocytes in their epithelium. In the nails, psoriasis can manifest in the forms of pitting nail, oil spot, and onychodystrophy. In the tounge, it can manifest as geographic tongue. ${ }^{4}$ Pitting nail is the detachment of some or all parts of the nail from the nail bed. Oil spot is a reddish-yellow discoloration under the nail that extends in the direction of the hyponicium. Onychodystrophy is the destruction of nail's morphology.14 Geographic tongue is the partial thickening of some parts of the tongue surface which results in islands-like formation..$^{12}$ In this study, $29.6 \%$ of the cases had nail involvement while $12.4 \%$ had tongue involvement.

Out of the 162 who were clinically diagnosed psoriasis, 46 had undergone biopsy. After biopsy, 33 cases were histopathologically confirmed as psoriasis while the other 13 were confirmed as no psoriasis. The histopathological types of the 13 cases were subcorneal pustular dermatosis (2), spongiotic dermatitis (2), pemphigous (2), psoriasiform reactions (2), pityriasis rosea (1), pityriasis chronic lichenoides (1), pityriasis rubra pilaris (1), lenticular hyperkeratosis (1), and neurodermatitis (1). On the other hand, there were 7 cases which had other diagnosis, but then were histopathologically confirmed as psoriasis. These 7 were initially diagnosed as tuberculosis cutis (2), pityriasis rosea (2), syphilis (1), seborrheic dermatitis (1), and leprosy (1). In this case, hyperkeratosis was found in $90 \%$ of all cases. This was similar to a previous study by Mehta et al. ${ }^{15}$ $(82 \%)$. The high rate of hyperkeratosis with parakeratosis in psoriasis patients conforms with the inflammatory pattern of psoriasis which is dominated by T-cells. Inflammatory cells, especially T-cells, will produce cytokines 
(IFN- $\gamma$, IL-2, IL-6, and TNF- $\alpha$ ) and growth factors which will induce proliferation of keratinocyte. ${ }^{3,4,13}$ These inflammatory factors are also the reason to the high rate of rete ridge elongation and/or acanthosis (98\%), which was congruent to a study by Moorchung et al. ${ }^{6}(100 \%)$. Keratinocyte hyperproliferation pushes the basal membrane and the dermis vertically, which is often described like a test tube in its rack. $^{6}$ The inflammatory pattern of psoriasis also causes neutrophil infiltration into the skin tissue. ${ }^{16}$ Neutrophils in the stratum corneum are called Munro's microabcess while those located in the stratum spinosum are called Kogoj's micropustules. ${ }^{1}$ In this study, Munro's microabcess and/ or Kogoj's micropustules were observed in $88 \%$ of all cases. This conformed to a previous study by Kassi et al. ${ }^{17}$ (90.9\%), but was higher than the finding of Mehta et al. ${ }^{15}$ $(62.3 \%)$. The high proportion of cases with the presence of Munro's microabcess and/or Kogoj's micropustules was probably caused by the large proportion of generalized pustular psoriasis and erythrodermic psoriasis cases, which are the psoriasis types with a pattern of inflammation that is activated by infections, unprescribed corticosteroid use, and irrational use of cream medication. ${ }^{1,11}$ Stratum granulosum can appear thinner or absent in psoriasis cases due to the high rate of keratinocyte proliferation which disrupt the process of keratinocyte differentiation into stratum granulosum. ${ }^{1}$ Thinning or absent stratum granulosum was observed in 15 cases $(37 \%)$, lower than what is reported by Mehta et al. ${ }^{15}$ (83.6\%). Thinning or absent stratum granulosum was also the reason why bleeding can readily happen if the scaly skin on psoriatic lesion is removed, as supported by the low proportion of Auspitz sign in this study. As previously explained, there is new formation of blood vessels that are fragile, tortuous, and dilated.6 In this study, the dilatation of blood vessels were observed in $75 \%$ of the cases, which was comparable to the studies by Mehta et al. ${ }^{15}(86.9 \%)$ and Kassi et al. ${ }^{17}(63.6 \%)$. There were some missing status in the histopathological medical records, namely: $4(10 \%)$ had missing information on hyperkeratosis with parakeratosis, $1(2 \%)$ had missing information on rete ridge elongation and/or acanthosis, 4 (10\%) had missing information on Munro's microabcess and/or Kogoj's micropustules, 25 (63\%) had missing information on the thinning or absence of stratum granulosum, and 10 (25\%) had missing information on dilated blood vessels.
The conclusion of this study was that there were 162 cases with clinical diagnosis of psoriasis, with reddish plaques and silvery scales as the most frequent characteristics (98.8\% and 95.7\% respectively). Meanwhile, out of the 40 cases that were histopathologically diagnosed psoriasis, the most prevalent characteristics were rete ridge elongation and/or acanthosis (98\%) and hyperkeratosis with parakeratosis $(90 \%)$.

One limitation of this study was the presence of medical records with incomplete or missing data in the Department of Dermatology and Venereology and the Department of Pathological Anatomy Dr. Hasan Sadikin General Hospital Bandung.

Based on the study, the author recommends histopathological examinations to be performed on atypical psoriatic lesions, as such lesions had histopathological diagnosis that was different from the clinical diagnosis. Another recommendation is that the patient examination and medical record writing, both clinical and histopathological, should be performed more extensively and systematically. Lastly, an integrated information system should be implemented in Dr. Hasan Sadikin General Hospital as it will be of great use in cases that involve multilple departments.

\section{References}

1. Gudjonsson JE, Elder JT. Psoriasis. In: Wolff L, Goldsmith LA, Katz SI, Gilchrest BA, Paller AS, Leffell DJ, editors. Fitzpatrick's dermatology in general medicine. 7th ed. New York: The McGraw-Hill; 2008.169-93.

2. Szczerkowska-Dobosz A, Placek W, Szczerkowska Z, Roszkiewicz J. The age of onset of psoriasis and the relationship to clinical presentation of psoriasis: study of 404 patients from northern Poland. Int J Dermatol. 2014;53(8):e367-8.

3. Krueger JG, Bowcock A. Psoriasis pathophysiology: current concepts of pathogenesis. Ann Rheum Dis. 2005;64 (Suppl2):S30-6.

4. Griffiths CE, Barker JN. Pathogenesis and clinical features of psoriasis. Lancet. 2007;370(9583):263-71.

5. Sabri AA, Qayyum MA. Psoriasis with Auspitz sign. CMAJ. 2006;175(1):31.

6. Moorchung N, Khullar J, Mani N, Chatterjee M, Vasudevan B, Tripathi T. A study of various histopathological features and their relevance in pathogenesis of psoriasis. Indian J Dermatol. 2013;58(4):294-8.

7. Stuart P, Malick F, Nair RP, Henseler T, Lim 
HW, Jenisch S, et al. Analysis of phenotypic variation in psoriasis as a function of age at onset and family history. Arch Dermatol Res. 2002;294(5):207-13.

8. Altobelli E, Petrocelli R, Marziliano C, Fargnoli MC, Maccarone M, Chimenti $\mathrm{S}$, et al. Family history of psoriasis and age at disease onset in Italian patients with psoriasis. Br J Dermatol. 2007;156(6):1400-1.

9. Jin Y, Yang S, Zhang F, Kong Y, Xiao F, Hou $Y$, et al. Combined effects of HLA-Cw6 and cigarette smoking in psoriasis vulgaris: a hospital-based case-control study in China. J Eur Acad Dermatol Venereol. 2009;23(2):132-7.

10. Augustin, Reich K, Glaeske G, Schaefer I, Radtke M. Co-morbidity and age-related prevalence of psoriasis: Analysis of health insurance data in Germany. Acta Derm Venereol. 2010;90(2):147-51.

11. Brenner M, Molin S, Ruebsam K, Weisenseel P, Ruzicka T, Prinz JC. Generalized pustular psoriasis induced by systemic glucocorticosteroids: four cases and recommendations for treatment. Br J Dermatol. 2009;161(4):964-6.
12. Dorland WAN. Dorland's illustrated medical dictionary. 28th ed. Philadelphia: Saunders Elsevier; 2009.

13. Purnak T, Purnak S. The inflammatory markers in psoriasis. Br J Dermatol. 2012;167(2):457-8.

14. Tan ES, Chong WS, Tey HL. Nail psoriasis: a review. Am J Clin Dermatol. 2012;13(6):375-88.

15. Mehta S, Singal A, Singh N, Bhattacharya SN. A study of clinicohistopathological correlation in patients of psoriasis and psoriasiform dermatitis. Indian J Dermatol Venereol Leprol. 2009;75(1):100.

16. Rocha-Pereira P, Santos-Silva A, Rebelo I, Figueiredo A, Quintanilha A, Teixeira F. The inflammatory response in mild and in severe psoriasis. Br J Dermatol. 2004;150(5):917-28.

17. Kassi K, Mienwoley OA, Kouyate M, Koui S, Kouassi KA. Severe skin forms of psoriasis in black africans: epidemiological, clinical, and histological aspects related to 56 cases. Autoimmune Dis. [Online Journal] 2013 [cited 2014 December 15]. Available from: www.hindawi.com/journals/ ad/2013/561032. 